\title{
Improvements on the present theoretical understanding of oc- tupole correlations
}

\author{
L.M. Robledo ${ }^{1, a}$ \\ ${ }^{1}$ Dep. Física Teórica, Universidad Autónoma de Madrid, E-28049 Madrid, Spain
}

\begin{abstract}
Some intriguing results, obtained in a recent survey of octupole properties for all even-even nuclei, are reanalyzed in order to understand the origin of the strong disagreement with experimental data and/or the strange behaviours observed. The limitations of the rotational formula to describe E1 and E3 transition strengths are discussed as well as the role played by octupole-quadrupole coupling in some specific nuclei.
\end{abstract}

\section{Introduction}

The properties of low lying collective negative parity states - including excitation energies and transition strengths - are strongly linked to the octupole deformation properties of the nucleus [1]. Octupole deformation can be static, when the nuclear wave function breaks the reflection symmetry or dynamic when it preserves the symmetry but quantum fluctuations involving octupole shapes are relevant [2]. A sound theoretical description encompassing both regimes is provided by the generator coordinate method (GCM) with the octupole deformation as generating coordinate [3]. Recently, such kind of calculations [4] have been performed for a set of 818 even-even nuclei and several variants of the Gogny interaction. Results for the excitation energy of the lowest lying collective negative parity state and the E1 and E3 transition strengths to the ground state have been computed and compared to experimental data. Several systematic inconsistencies in the comparison can be noticed, including a systematic overestimation of excitation energies and an underestimation of the E3 transition strengths. Also some "wild" results like the case of ${ }^{64} \mathrm{Zn}$ were worth investigating.

\section{Theoretical tools}

Except in very specific cases, most of the theoretical descriptions of low energy nuclear structure are based on an underlying mean field description that is supplemented afterwards by other many body techniques like the Random Phase Approximation (RPA), symmetry restoration (SR) and the generator coordinate method (GCM). In our case, we start by generating a set of mean field Hartree-FockBogoliubov (HFB) wave functions labeled by the values of the axially symmetric octupole moment $Q_{30}$. The other deformation parameters are chosen self-consistently by the interaction in order to minimize the energy. The HFB states $\left|\varphi\left(\beta_{3, i}\right)\right\rangle$ labelled by the discrete variable $\beta_{3, i}$ are used as a restricted basis to expand octupole correlated wave functions $\left|\Psi_{\sigma}\right\rangle=\sum_{\beta_{3, i}} f_{\sigma}\left(\beta_{3, i}\right)\left|\varphi\left(\beta_{3, i}\right)\right\rangle$ This ansatz

\footnotetext{
ae-mail: luis.robledo@uam.es
} 
restores the broken reflection symmetry by relating the parity $\pi_{\sigma}$ of $f_{\sigma}\left(-\beta_{3, i}\right)=\pi_{\sigma} f_{\sigma}\left(\beta_{3, i}\right)$ to the one of $\left|\Psi_{\sigma}\right\rangle$. The $f_{\sigma}$ amplitudes are determined as to minimize the energy by solving the Hill-Wheeler secular equation [3]

$$
\sum_{\beta_{3, j}}\left\langle\varphi\left(\beta_{3, i}\right)|\hat{H}| \varphi\left(\beta_{3, j}\right)\right\rangle f_{\sigma}\left(\beta_{3, j}\right)=E_{\sigma} \sum_{\beta_{3, j}}\left\langle\varphi\left(\beta_{3, i}\right) \mid \varphi\left(\beta_{3, j}\right)\right\rangle f_{\sigma}\left(\beta_{3, j}\right)
$$

Overlaps of observables $\hat{O}$ are computed the usual way

$$
\left\langle\Psi_{\sigma}|\hat{O}| \Psi_{\sigma^{\prime}}\right\rangle=\sum_{\beta_{3, i}, \beta_{3, j}} f_{\sigma}^{*}\left(\beta_{3, i}\right) f_{\sigma^{\prime}}\left(\beta_{3, j}\right)\left\langle\varphi\left(\beta_{3, i}\right)|\hat{O}| \varphi\left(\beta_{3, j}\right)\right\rangle
$$

Finally, transition strengths are computed assuming the validity of the rotational formula not only for mean values but also for overlaps. For instance, assuming that $\left|\Psi_{1}\right\rangle$ and $\left|\Psi_{0}\right\rangle$ are the intrinsic states associated to the $3^{-}$and $0^{+}$states, the $\mathrm{E} 3$ strength is given by $B\left(E 3,3^{-} \rightarrow 0^{+}\right)=e^{2} /(4 \pi)\left|\left\langle\Psi_{1}\left|\hat{Q}_{3}\right| \Psi_{0}\right\rangle\right|^{2}$. For the energies of the physical states $J^{\pi}$ we add the rotational correction $J(J+1) /\left(2 \mathcal{J}_{T V}\right)$ involving the Thouless-Valatin (TV) moment of inertia computed in the cranking approximation. Usually, the correction for the $1^{-}$and $3^{-}$states is small compared to the octupole splitting.

The $f$ can not be used as probability amplitudes because the set of HFB wave functions is not orthogonal. The derived quantities $g_{\sigma}\left(\beta_{3, i}\right)=\sum_{\beta_{3, j}}\left\langle\varphi\left(\beta_{3, i}\right) \mid \varphi\left(\beta_{3, j}\right)\right\rangle^{1 / 2} f_{\sigma}\left(\beta_{3, j}\right)$ have to be used instead. Here, the square root has to be understood as the square root of the norm matrix.

The GCM method can be easily extended to handle the coupling of several degrees of freedom, like quadrupole and octupole collective coordinates (see below). In our survey of [4] the above method is implemented with the the three standard parametrization (D1S, D1N and D1M) of the Gogny force. The Gogny force has been chosen because of its reputation in describing many different phenomena all over the nuclear chart. The phenomenological density dependent term present in the Gogny force has to be generalized in order to compute the Hamiltonian overlaps. Consistency requirements [5] and difficulties [6] force the use of the "overlap density" prescription.

\section{Discussion of results}

\subsection{Excitation energies}

Excitation energies of negative parity states are predicted [4] to be systematically higher than experimental data. A hint on the origin of the discrepancies is the good agreement with experiment for the $3^{-}$excitation energy of ${ }^{208} \mathrm{~Pb}$ computed with RPA and the Gogny force [7]. In the GCM framework the 4.0 MeV obtained is well above the experimental value of $2.6 \mathrm{MeV}$. On the other hand, the RPA is the small amplitude limit of the GCM [3] provided all possible degrees of freedom are considered in the GCM. Therefore, it is likely that the excitation energies could be brought closer to experiment by including other degrees of freedom in addition to the octupole one. Exploratory work on the quadrupole-octupole coupling is discussed in $[8,9]$ and also below.

\subsection{The nucleus ${ }^{64} \mathrm{Zn}$}

The nucleus ${ }^{64} \mathrm{Zn}$ is a typical case showing strong discrepancies with experiment. The predicted excitation energy for the intrinsic negative parity state is $7.19 \mathrm{MeV}$ to be compared with the experimental value of $2.998 \mathrm{MeV}$ for the $3^{-}$state. In this nucleus the rotational correction for the prolate minimum for the $3^{-}$state amounts to $0.920 \mathrm{MeV}$ which is much smaller than $7.19 \mathrm{MeV}$ but still significant and 
goes in the opposite direction. The experimental E3 transition strength of $20 \pm 3$ W.u. [10] is far from the wildly fluctuating values obtained with different interactions and different methods. For instance, parity projection with the Gogny D1S gives a E3 strength of $10^{-4}$ W.u. A closer look at the HFB wave functions reveals that the quadrupole moment varies from oblate to prolate as a function of the octupole moment. This is a clear indication of the relevance of the quadrupole-octupole coupling. We have carried out GCM calculations for ${ }^{64} \mathrm{Zn}$ with the axially symmetric quadrupole and octupole moments as generating coordinates. The HFB energy obtained with Gogny D1S is depicted in the left hand side panel of Fig 1. We observe two minima, one (absolute) oblate and the other prolate lying very close in energy. As the octupole moment increases, the energy of the prolate configuration goes below the oblate one. The GCM calculation produces probability amplitudes plotted in the middle and right hand side panels of the figure for the ground state and first negative parity state, respectively. As a consequence of the octupole dynamics, the ground state becomes prolate but with a large
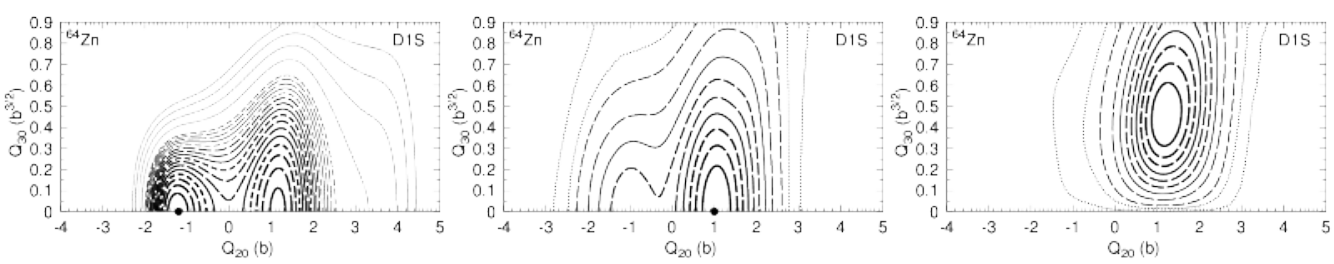

Figure 1. From left to right, contour plots of the HFB energy and the amplitudes $\left|g_{\sigma}\left(Q_{20}, Q_{30}\right)\right|^{2}$ for the ground state (middle) and first negative parity state (right panel).

oblate component. On the other hand, the negative parity state is prolate with a probability amplitude concentrated in the prolate side.

Table 1. Comparison of experimental data for ${ }^{64} \mathrm{Zr}$ [10] with the results of different calculations: PP-RVAP is for parity projection [4], GCM-Q3 is for the one dimensional GCM calculation of [4] and GCM-Q2-Q3 is for the present calculation. The rotational energy correction (of the order of $900 \mathrm{keV}$ ) is not considered for the predicted excitation energies.

\begin{tabular}{ccccc}
\hline & Exp & PP-RVAP & GCM-Q3 & GCM-Q2-Q3 \\
\hline$E_{3^{-}}(\mathrm{MeV})$ & 2.998 & 4.10 & 7.19 & 4.20 \\
\hline B(E3) W.u. & $20 \pm 3$ & $10^{-4}$ & 2.83 & 6.75 \\
\hline
\end{tabular}

The results for the $Q_{20}-Q_{30}$ calculations are summarized in Table 1. As compared to the GCM-Q3 calculation, the quadrupole-octupole coupling reduces the excitation energy from 7.19 to $4.20 \mathrm{MeV}$ and increases the transition strength by a factor of 2 improving the agreement with experimental data. The nucleus ${ }^{64} \mathrm{Zn}$ is predicted to be $\gamma$ soft by many interactions. Therefore the coupling between the $\gamma$ degree of freedom and the $K \neq 0$ components of the octupole operator could affect the results.

\subsection{E3 strengths}

Another relevant deficiency of the results of Ref [4] is the systematic underestimation of E3 transition strengths for nuclei with a not well deformed ground state. The problem can be traced back to the validity of the rotational formula used to obtain the transition strengths from the multipole moments of the intrinsic states [3]. In a study using wave functions projected to good angular momentum [11] we showed that the rotational formula breaks badly down for spherical or near spherical HFB 
Table 2. B(E3) transition strengths in W.u. for the different approaches considered. See Table 1 for notation. In all the cases, the suffix AMP represents Angular Momentum Projected calculations. The last column is the quadrupole deformation parameter $\beta_{2}$ for the ground state.

\begin{tabular}{ccccccc}
\hline & Exp & PP-RVAP & PP-RVAP-AMP & GCM-Q3 & GCM-Q3-AMP & $\beta_{2}$ (GS) \\
\hline${ }^{20} \mathrm{Ne}$ & 13 & 9.73 & 12.78 & 12.46 & 12.31 & 0.66 \\
\hline${ }^{64} \mathrm{Zn}$ & $20 \pm 3$ & $10^{-4}$ & 0.6 & 2.83 & 5.75 & -0.21 \\
\hline${ }^{158} \mathrm{Gd}$ & 12 & 9.43 & 10.29 & 13.73 & 12.64 & 0.34 \\
\hline${ }^{208} \mathrm{~Pb}$ & 34 & 7.07 & 23.06 & 7.67 & 21.93 & 0 \\
\hline${ }^{224} \mathrm{Ra}$ & $42 \pm 3$ & 71.39 & 71.39 & 70.16 & 61.51 & 0.18 \\
\hline
\end{tabular}

configurations. The discrepancies could be as large as a factor 7 (the multiplicity of $\mathrm{L}=3$ ) for the E3 strength when computed in the Parity Projection (PP) RVAP framework. We have extended those PP-RVAP calculations to consider the $Q_{30}$ GCM wave functions of [4] as intrinsic states. Preliminary results in a few emblematic cases are shown in Table 2. It has to be mentioned that the $f$ amplitudes of the GCM states should be computed in a framework including angular momentum projection (AMP). Whether the projected amplitudes reduce or magnify the impact of AMP on the transition strength remains to be seen.

We observe that in most of the cases, the net effect of projection onto good angular momentum - in order to obtain the E3 transition strength - is to bring the theoretical predictions much closer to the experimental data. The effect is important in the shape coexisting ${ }^{64} \mathrm{Zn}$ and the spherical ${ }^{208} \mathrm{~Pb}$ nuclei where the E3 strength increases by a factor of 2 or 3. On the other hand in the weakly deformed ${ }^{224} \mathrm{Ra}$, projection reduces the transition strength given by the rotational formula. This is a clue that the rotational formula is even more difficult to justify when correlated wave functions are used. Finally, it is noticed that the ratio projected/unprojected W(E3) is close to one for those nuclei with a well deformed ground state (see last column of Table 2) and can be as large as a factor 3 otherwise. The results are encouraging and work is in progress to both extend the GCM-Q3 projected calculation of the $\mathrm{W}(\mathrm{E} 3)$ to all the even-even nuclei considered in [4] and to include it in GCM-Q2-Q3 calculations.

\section{Acknowledgments}

Work supported in part by MICINN grants Nos. FPA2009-08958, FIS2009-07277, and by the Consolider-Ingenio program MULTIDARK CSD2009-00064.

\section{References}

[1] P. A. Butler and W. Nazarewicz, Rev. Mod. Phys. 68, 349 (1996)

[2] L. P. Gaffney et al, Nature 497, 199 (2013)

[3] P. Ring and P. Schuck, "The Nuclear Many-Body Problem", (Springer, NY, 1980)

[4] L. M. Robledo and G. F. Bertsch, Phys. Rev. C 84, 054302 (2011)

[5] L.M. Robledo, Intl. J. of Mod. Phys. E 16, 337 (2007)

[6] L.M. Robledo, J. Phys. G 37, 064020 (2010)

[7] S. Péru, J.F. Berger, and P.F. Bortignon, Eur. Phys. J. A 26, 25 (2005)

[8] L.M. Robledo, J.L. Egido, B. Nerlo-Pomorska and K. Pomorski, Phys. Lett. B 201, 409 (1988)

[9] R. Rodriguez-Guzman, L. M. Robledo and P. Sarriguren, Phys. Rev. C 86, 034336 (2012)

[10] T. Kibédi and R. Spear, Atomic Data and Nuclear Data Tables 80, 35 (2002)

[11] L. M. Robledo and G. F. Bertsch, Phys. Rev. C 86, 054306 (2012) 